\title{
COVID-19: dental aerosol contamination in open plan dental clinics and future implications
}

\section{Kamran Ali ${ }^{1}$ and Mahwish Raja ${ }^{2}$}

\section{A commentary on}

\section{Holliday R, Allison J R, Currie C C et al.}

Evaluating contaminated dental aerosol and splatter in an open plan clinic environment: Implications for the COVID-19 pandemic. J Dent 2021; 105: 103565.

\section{Abstract}

Study design An experimental study design was used to investigate the spread of splatter/aerosol during simulated dental procedures on a mannequin in open plan clinic and dental clinical teaching laboratory settings. All experiments were based on crown preparation of an artificial maxillary central incisor using a high-speed air turbine over a ten-minute period. Fluorescein dye was introduced into the irrigation system of the handpiece (model 1) and the mannequin's mouth was used to simulate salivary flow (model 2) under varying experimental conditions (suction flow rate, cross ventilation and exposure time). Six experiments were conducted in the open plan clinic while three experiments were undertaken in the clinical laboratory. Customised rigs with collection platforms consisting of filter papers were placed in open plan bays and adjacent walkways. Samples were also collected from a $400 \mathrm{~cm}^{2}$ area in each of the eight adjacent bays. Time course experiments repeated the same procedures on three occasions in a clinical laboratory and utilised a rig with eight four-metre rods supporting collection platforms around a dental mannequin. The distribution of fluorescein dye was analysed by fluoroscopy and spectrofluorometry.

Results Contamination levels showed variations under different experimental conditions. In the absence of suction and cross ventilation, contamination was observed at large distances. Use of suction reduced contamination in the operating bay by $53 \%$ and $81.83 \%$, while cross ventilation reduced contamination in adjacent and distant areas by $80-89 \%$. Minimal contamination was detected at a distance of $>5 \mathrm{~m}$ from the operating bay, with the use of medium-volume suction demonstrating that 1.5 -metre-high partitions with open fronts limit $99.99 \%$ of splatter from aerosol generating procedures (AGPs) to the operating bay. Minimal additional aerosol contamination was detected ten minutes after the procedure.

Conclusions Contamination from dental AGPS has the potential to contaminate distant sites in open plan clinics. Risk of cross infection is small if the bays are $>5 \mathrm{~m}$ apart and contamination can be minimised with the use of suction and cross ventilation.

\section{Practice points}

- The risks of COVID-19 infection with bioaerosols generated during AGPs in open plan clinics may be minimised with the use of PPE, intraoral suction, rubber dam isolation (when appropriate), speed-increasing handpieces, disinfection of the clinical environment and appropriate air exchange systems.

- Dental institutions should continue to implement standard operating procedures including a fallow period based on national/international guidelines.

- Further research may help generate more evidence to reduce fallow period in open plan dental clinics.

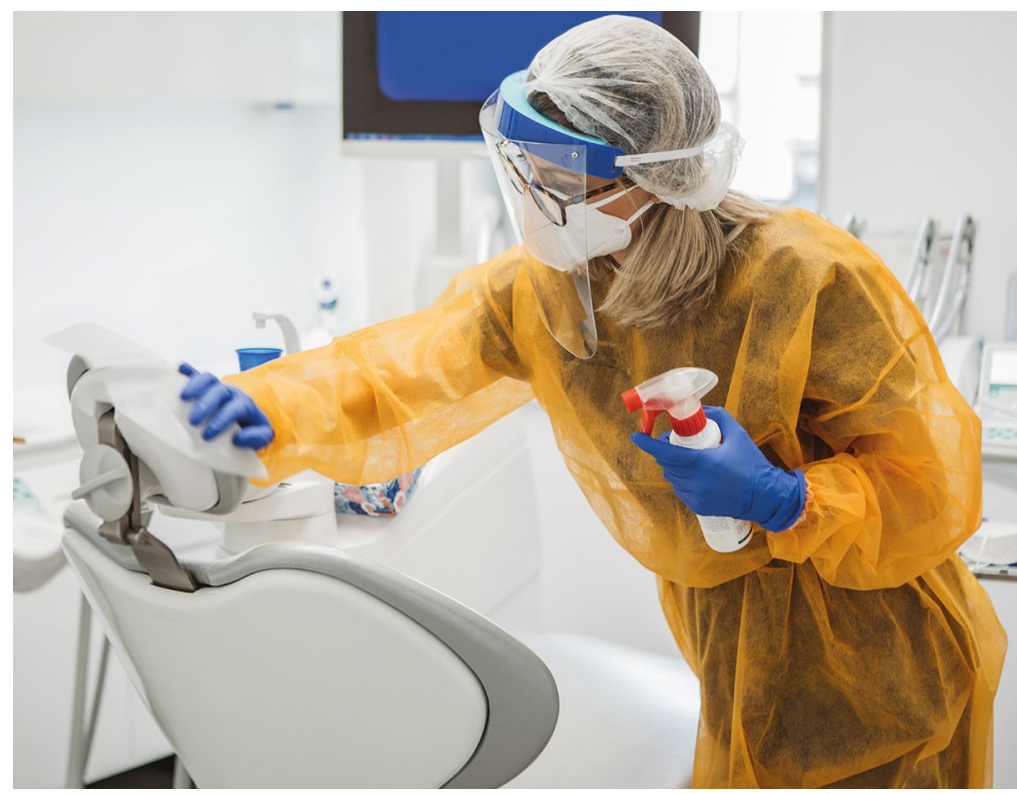

\section{Commentary}

The coronavirus disease (COVID-19) caused by the novel coronavirus (SARS-CoV-2) has had the most remarkable impact on delivery of clinical dental services and education in living memory. Following an initial period of fear and anxieties at the start of the pandemic, dental practices and institutions across the globe are now back in operation, albeit at lower efficiency levels due to additional precautions mandated by professional guidelines. One of the major concerns with clinical dentistry relates to the risks of SARS-CoV-2 contamination during aerosol generating procedures (AGPs). The virus is present in nasopharyngeal secretions, saliva and blood, and bioaerosols generated in the dental clinical environment may cause cross infection through inhalation, contact with eyes and contaminated work surfaces. ${ }^{1}$ Although there is no direct evidence to substantiate the spread of SARS-CoV-2 through dental aerosols, guidelines continue to advise additional precautions to reduce the risk of cross infection. ${ }^{2}$

The COVID-19 pandemic has posed unique challenges for dental organisations with open plan clinics, particularly dental 
hospitals and universities. ${ }^{3}$ Dental teaching institutions have had to adopt a wide range of risk mitigation strategies for AGPs in open plan clinics since the start of the pandemic. These include: a combination of physical and temporal separation measures; compartmentalisation of open plan clinics to create self-contained pods for AGPs; use of high-volume intraoral and extraoral suction devices; use of speed-increasing handpieces; and installation of air exchange systems. ${ }^{4}$ In any case, these cross-infection control measures have impacted adversely on dental services, with significant resource and logistic implications for the providers. Clinical training of dental students is usually provided in open plan clinics. It has been immensely difficult for students to achieve their annual clinical targets since the start of the pandemic, which has impacted on their sign off for finals, and it continues to be a huge challenge for dental schools globally.

The current study by Holliday et al. provides new evidence regarding the pattern of aerosol spread in simulated open plan dental clinical environments. The authors have used a variety of standardised experimental conditions to quantify aerosol spread and its impact on fallow time for dental AGPs. The findings of this study are corroborated by a recent study by Ehtezazi et al. ${ }^{5}$ The evidence emerging from these studies indicates that with appropriate use of personal protective equipment (PPE), suction devices, air exchange systems and disinfection of the clinical environment, it may be possible to reduce the fallow time for AGPs to ten minutes in open plan clinics. Further studies in realtime open plan clinical settings are required to substantiate these findings to inform professional guidelines.
The ongoing COVID-19 vaccination campaigns provide a glimmer of hope that some degree of normality might be restored in the future. However, the rapid emergence of mutant strains of SARS-CoV-2 and lack of clarity regarding the longevity of immune protection imparted by vaccines add to the existing uncertainties related to COVID-19. In addition, fake propaganda on social media platforms fuelled by conspiracy theories is a barrier to widespread uptake of vaccines, particularly among some sections of society. ${ }^{6}$ With the third wave of COVID-19 already looming, it seems likely that COVID-19 is here to stay in some form and the dental profession must continue to adapt in light of emerging scientific evidence.

\section{Author affiliation}

${ }^{1}$ University of Plymouth Peninsula Dental School, ${ }^{2}$ Mahwish Raja, Newcastle University, UK.

Correspondence to: Kamran Ali

\section{References}

1. Peng X, Xu X, Li Y, Cheng L, Zhou X, Ren B. Transmission routes of 2019-nCoV and controls in dental practice. Int J Oral Sci 2020; 12: 9.

2. Epstein J B, Chow K, Mathias R. Dental procedure aerosols and COVID-19. Lancet Infect Dis 2021; DOI: 10.1016/S1473-3099(20)30636-8.

3. Sukumar S, Dracopoulos S A, Martin F E. Dental education in the time of SARS CoV-2. Eur J Dent Educ 2021; 25: 325-331.

4. Witton R, McColl E, Tredwin C. Students' return to clinic. Br Dent J 2021; 230: 3.

5. Ehtezazi T, Evans D G, Jenkinson I D et al. SARS-CoV-2: characterisation and mitigation of risks associated with aerosol generating procedures in dental practices. Br Dent / 2021; DOI: 10.1038/s41415-020-2504-8.

6. Rzymski, P, Borkowski, L, Drąg, M et al. The Strategies to Support the COVID-19 Vaccination with Evidence-Based Communication and Tackling Misinformation. Vaccines (Basel) 2021; 9: 109.

Evidence-Based Dentistry (2021) 22, 54-55.

https://doi.org/ 10.1038/s41432-021-0168-0 